\title{
TORSIONAL STIFFNESS AS AN INFLUENCE PARAMETER ON ARCH STABILITY
}

\author{
PHILIPPE VAN BOGAERT, GILLES VAN STAEN, and HANS DE BACKER \\ Dept of Civil Engineering, Ghent University, Ghent, Belgium
}

\begin{abstract}
Arch bridge springs can be connected to concrete abutments either by prestressing bars or by connectors. In both options, the torsional stiffness is substantially reduced, compared to the full arch cross sectional area. The influence of this lack of torsional stiffness on arch buckling is being researched, both numerically and experimentally. To reduce any residual stress during tests, wooden rods that simulate the arch were submerged in water and subsequently bent to the desired shape. Imperfections of the arch samples are measured. Two unequal concentrated loads are applied to the samples, thus simulating the effect of movable loads across half of the arch span. During loading, lateral deflections were measured until elastic buckling occurred. The simulation of more flexible rotation of the springs required replacing the cross section by thin equivalent side plates. Since all parameters have not been isolated, the results are limited yet. However, comparing the failure load of similar conditions, the reduction of torsion stiffness by $81.48 \%$ reduces the failure load by $26.3 \%$. This indicates that total prevention of axial rotation may not be imperative for arch bridges.
\end{abstract}

Keywords: Arch spring, Arch buckling load, Connections, Scaled tests, Nonlinear calculation.

\section{INTRODUCTION - ARCH SPRING CLAMPING}

The connection of springs to concrete abutments is of paramount importance for the load carrying capacity of arch bridges. At this location the various reactions from the bridge superstructure are transferred to the abutments. The most important reaction is the arch thrust force, together with the vertical reactions. Whether bending moments are transferred to the infrastructure at the arch springs, depends on the use of hinges or clamping. The fabrication of hinges certainly is more complicated than complete clamping of arch springs. In most cases, the concept of the arch spring connections corresponds to clamping and bending moment transfer.

A particular reaction is the torsion moment. Since the torsional capacity and stiffness of circular sections are rather high, large torsion clamping may be expected. However, experience mentioned in Van Bogaert (2013) has shown this torsion stiffness may be rather low, depending on the type of connection being used. Connections with prestressing bars may appear to be an excellent choice to materialize the clamping condition, but failed for torsion in at least the application mentioned. Therefore, the alternative of using connectors has been considered. In this case as well, the torsion stiffness proved to be smaller than expected as demonstrated by Van Bogaert et al. (2018). Because of this, a small number of tests was carried out and has given better insight in the issue. Both strip and headed stud connectors were used. However, both alternatives have demonstrated that excessive axial rotation of springs of arch bridges, connected to concrete abutments can hardly be avoided. Strip connectors show large flexibility, whereas the 
stiffer stud connectors still show 13-times less stiffness than the base tube. In addition, the connection with prestressing bars was also unable to prevent the axial rotation, due to torsion. Clearly, a wider experimental research may provide larger insight in the torsional clamping of steel tubes in concrete. In the present state, it may be recommendable for design not to consider complete rotational fixity of the arch bridge springs considered.

As a consequence, the objective of the present research was to determine to which extent torsion clamping influences the arch stability. Obviously, the stabilizing action of heavy end crossbeams or upper bracings is excluded and the most critical case of free standing arches has been considered.

\section{FREE STANDING ARCH}

The first approximation to obtain understanding of the importance of axial rotations consists in determining the elastic buckling modes and factors of a free standing arch. As the research was directed to test scaled models from balsa wood, a small structure was first verified. A free standing arch is the elementary form and will evidently allow to address the effect of a lack of torsion stiffness. With this simple model, there is no contribution to arch stability by an upper bracing or any stabilizing effect of hangers or bars connecting the arch to the bridge deck. This allows to eliminate all other effects and to concentrate on the single issue of the torsion stiffness of the arch spring. The lowest factor directly determines the elastic critical load and the arch reduced slenderness. Figure 1 left shows the buckling modes for a $500 \mathrm{~mm}$ span and $90 \mathrm{~mm}$ rise clamped arch with rectangular cross-section of $8 * 8 \mathrm{~mm}$, whereas the right figure shows the result for the same arch, the axial rotation being completely free. The load consists of 2 knife forces applied at $1 / 4$ and $3 / 4$ of the span length. A single concentrated load would result in a low buckling load and unprecise results. The critical section of the arch being at $1 / 4$ span length, two concentrated loads are used, the first being twice as large as the second, as shown in Figure 1 left. This introduces unequal bending and is closer to the real situation.

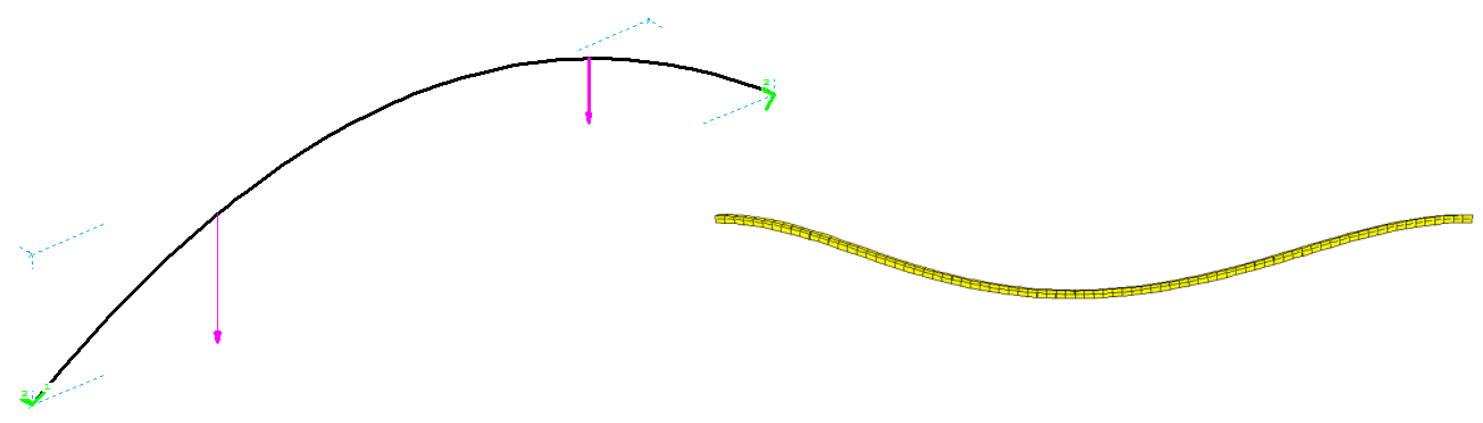

Figure 1. Elastic buckling mode clamped and free arch.

The elastic buckling load of the clamped arch exceeds the value for the free arch by $21.3 \%$ only. Both mode shapes are similar and correspond to lateral torsional buckling, as seen from above in Figure 1 right. Hence, the influence of torsional stiffness on the buckling load is moderate and the research should concentrate on lateral buckling.

Clearly, the above numerical model is inadequate to address the effect of imperfections, of material nonlinearity and of residual stress, all important factors, influencing the arch stability. Hence, in the following a 3-D continuous element has been used to simulate the arch conduct. The material law takes into account elastic behavior from $\varepsilon=0$ to $\varepsilon=\mathrm{f}_{\mathrm{m}} / \mathrm{E}$ and a short branch ending at the coordinates $\varepsilon=0.01 \sigma=1.1 \mathrm{f}_{\mathrm{m}}$ since according to EN 1995-1-1 wood has but a 
small post-yielding capacity. This is in accordance with data from suppliers. The values of $E$ in the test samples will be discussed later.

In addition, imperfections have been introduced in the numerical model, their shape corresponding to the fundamental mode shapes from Figure 1. It is believed that these correspond to the half wave, with a slightly prevented lateral rotation at the springs.

\section{TESTING OF SCALED SPECIMENS}

For the tests, rods of balsa wood, a commonly used model material, have been used. The cross section of each rod was $4 * 4 \mathrm{~mm}$. The rods were bent to obtain the curved arch shape. Since this introduces large residual stress, up to breaking of the rod, an alternative process was adopted. The rods were immersed in water for 45 minutes, bent by positioning them on a work floor and maintained in this position during drying. This process was repeated twice for each rod. Figure 2 shows the rods in the drying position.

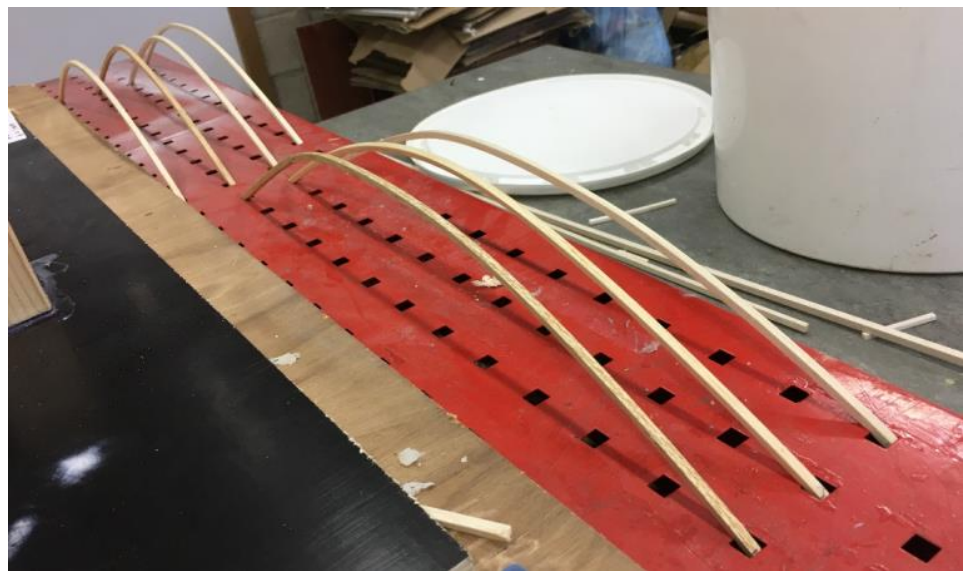

Figure 2. Wetting-drying and shaping of rods.

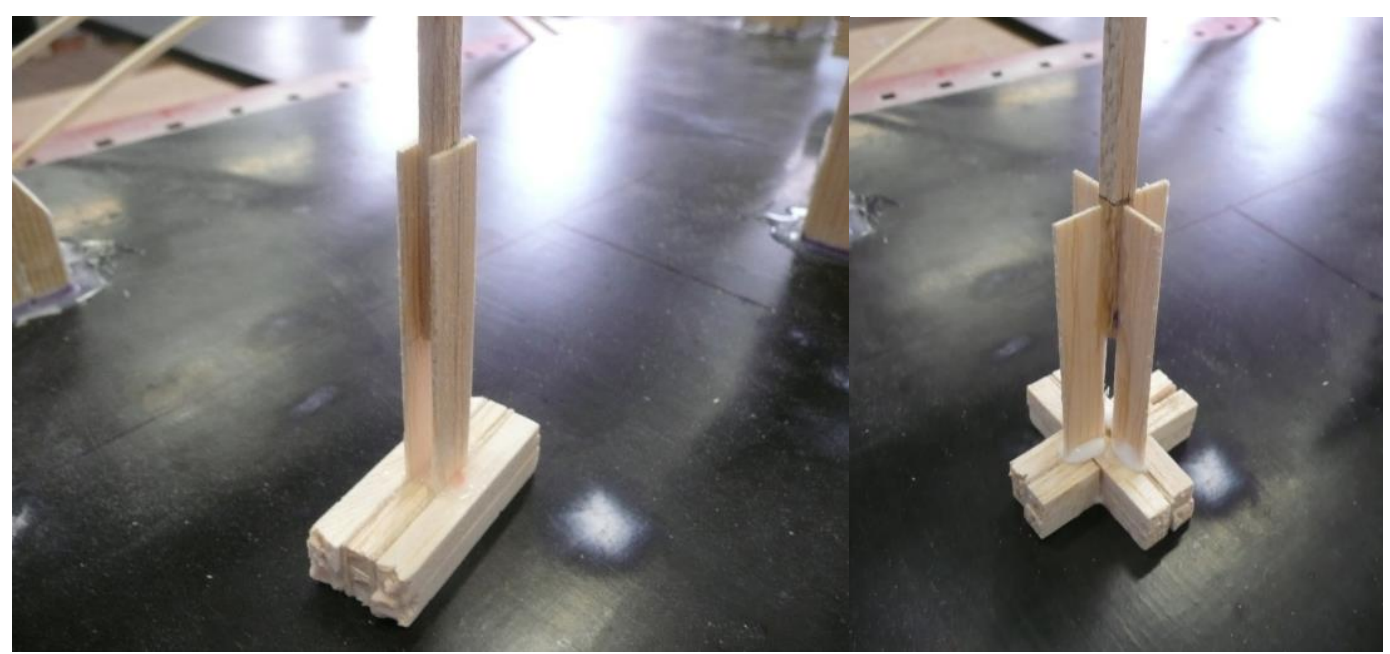

Figure 3. Materialization of weaker axial rotation stiffness (left) parallel plates (right) cross. 
Since the E-modulus of the wood shows no constant value and is changing during the wetting-drying process, for each rod the modulus has been measured. After drying the modulus has increased from its original value and varied between $6900 \mathrm{MPa}$ to $12611 \mathrm{MPa}$. This means every test value had to be compared to numerical results, using the experimental modulus.

The experimental simulation of arch springs to a fixed infrastructure for the torsion fixed condition does not rise a particular issue. However, a free axial rotation is virtually impossible to build. Hence, various ways of 'weakening' the arch springs for torsion have been considered. Torsion stiffness is reduced by replacing a plain section by open sections, either 2 parallel plates of $10^{*} 1 \mathrm{~mm}$ or 4 crossing thin plates, both shown in Figure 3 . Theoretically the parallel plate scheme has 0.185 torsion stiffness of the plain rod, whereas the cross scheme has 0.37 - times this value.

Figure 3 also shows the rod's end. The arch span of these types of connections has been slightly increased by the distance from the spring to halfway the weakened part. This would correspond to the concrete encasing of the arch spring.

In total 7 specimens of fixed arches have been tested, supplemented by 8 specimens with alternative spring cross sections, of which 5 of the plate type and 3 with cross sections. The test setup is shown in Figure 4, including the position of the eccentric loading via a small plank on which the $0.1 \mathrm{~kg}$ mass loads are added. During loading the lateral displacements of the arch crown are measured.

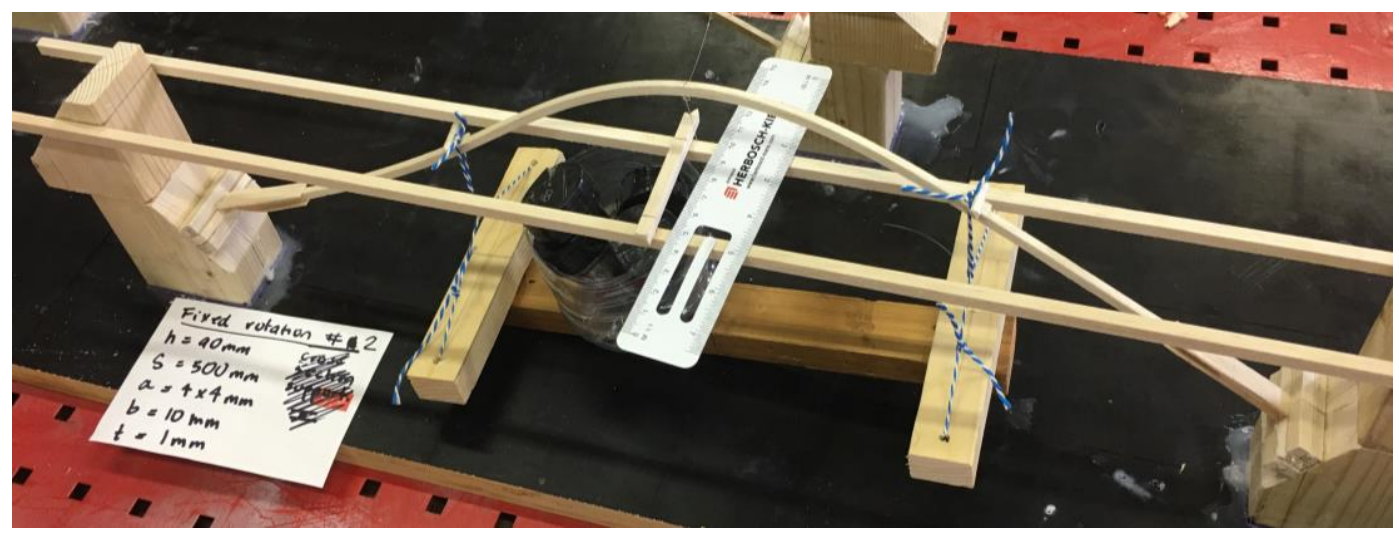

Figure 4. Test setup, loading and measurement of lateral displacement.

The lateral displacements allow assessing whether or if buckling is occurring. However, some of the specimens failed without previous displacement, due to failure of the wood. This attributes to the low plastic capacity of the wood and its heterogynous nature. Nevertheless, the curves of lateral displacement and applied load allow drawing of the Southwell-plot as described in William (1987) allows determining the experimental value of the buckling load.

\section{COMPARING RESULTS WITH SIMULATIONS}

Since the specimens showed 3 independent parameters: E-modulus, initial imperfection and the type of arch spring, it became difficult to isolate one of these parameters and clearly assess its influence on the arch failure load. In addition, the failure load itself needs to be defined. Because of the nonlinearity and the initial imperfection, a small amount of load already initiates lateral displacement. A clear bifurcation load does not exist. However, this was overcome by identifying the critical load to the value found from the Southwell plot. 
In Table 1 all tests are summarized, including the type of arch spring, E-modulus, initial imperfection, final collapse load and critical load. The last column of this table also mentions the failure load found from the nonlinear simulation with 3-D volume elements. The use of this type of elements, allowing simulating both geometric and material nonlinearity enables to reproduce exactly the physical models, in particular the geometry of the arch springs.

Table 1. Test results summarized.

\begin{tabular}{lccccc}
\hline Spring shape & E (MPa) & Imperfection (mm) & Failure load (N) & Southwell (N) & Numerical load (N) \\
\hline Fixed & 5554.42 & 0 & 12.83 & 13.49 & 18.07 \\
Fixed & 5554.42 & 1.75 & 14.09 & 14.37 & 18.07 \\
Fixed & 5554.42 & 5.5 & 8.20 & 7.25 & 18.12 \\
Fixed & 3123.78 & 0 & 7.22 & 7.47 & 10.16 \\
Fixed & 20243.64 & 0.5 & 9.18 & 9.53 & 65.85 \\
Fixed & 12611.39 & 3.25 & 7.22 & 7.73 & 41.07 \\
Fixed & 3123.78 & 0.5 & 4.28 & 5.26 & 10.16 \\
Plates & 5554.42 & 2.25 & 10.23 & 10.59 & 27.73 \\
Plates & 20243.64 & 3.25 & 9.93 & - & 101.10 \\
Plates & 20243.64 & 2.75 & 11.14 & 15.44 & 101.07 \\
Plates & 5554.42 & 1.5 & 8.20 & 11.77 & 27.72 \\
Plates & 3123.78 & 1.5 & 3.30 & 3.70 & 15.59 \\
Cross & 7681.42 & 0.25 & 12.12 & 13.41 & 39.72 \\
Cross & 7457.80 & 900 & 10.16 & 9.09 & 38.56 \\
Cross & 7837.27 & 2.25 & 8.20 & 14.55 & 40.54 \\
\hline
\end{tabular}

Numerical simulation results in 2 types of data. The first is a diagram of load versus lateral displacement, clearly showing the nonlinear character of calculations, as shown in Figure 5. In some cases, the lateral displacement changes sign while the load increases. The second results are stresses at each load increment. Figure 6 shows equivalent vonmises stresses of the arch for the plate connected spring and with $1 \mathrm{~mm}$ initial imperfection, immediately before collapse. Obviously, the largest stresses appear near the location of the largest of both loads and has reached the failure value.

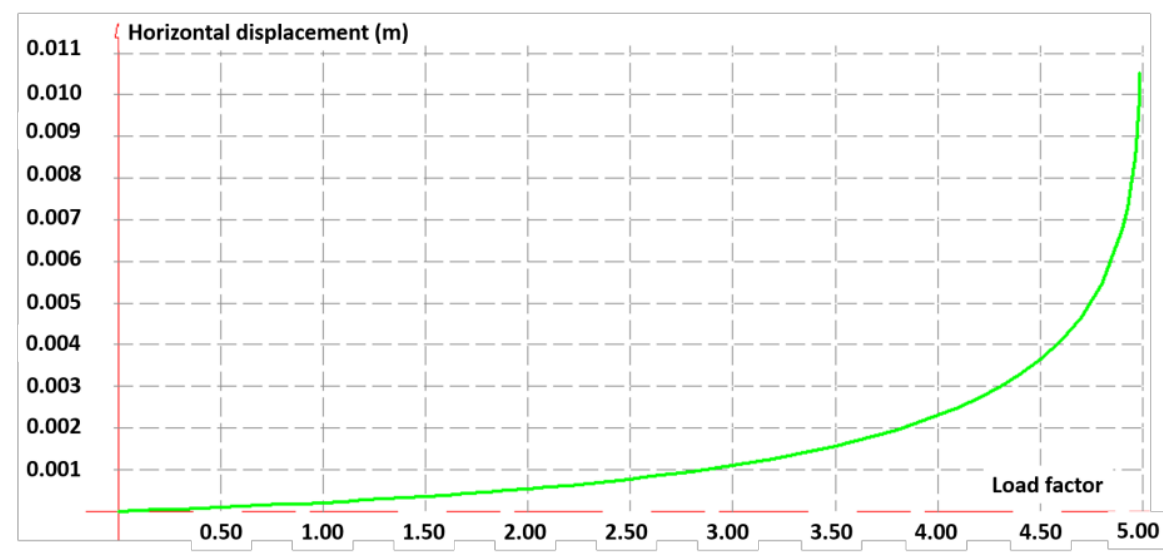

Figure 5. Load-displacement path numerical simulation.

The results have been categorized according to the parameters of large initial imperfection and of E-modulus. Clearly, identical combinations are not available and should require a considerably larger amount of specimens and tests. 
However, rows 2 and 11 of Table 1 are somehow similar, the E-modulus and the imperfection being similar. The failure load of the weaker arch spring decreases the failure load by $18.1 \%$. This is rather close to the $21.3 \%$ of the elastic beam prediction and applies to a reasonable amplitude of the imperfection. The numerically determined values overestimate the failure load, surely by adopting an excessive value of the unknown wood resistance and showing a similar gap between both arch spring conditions.

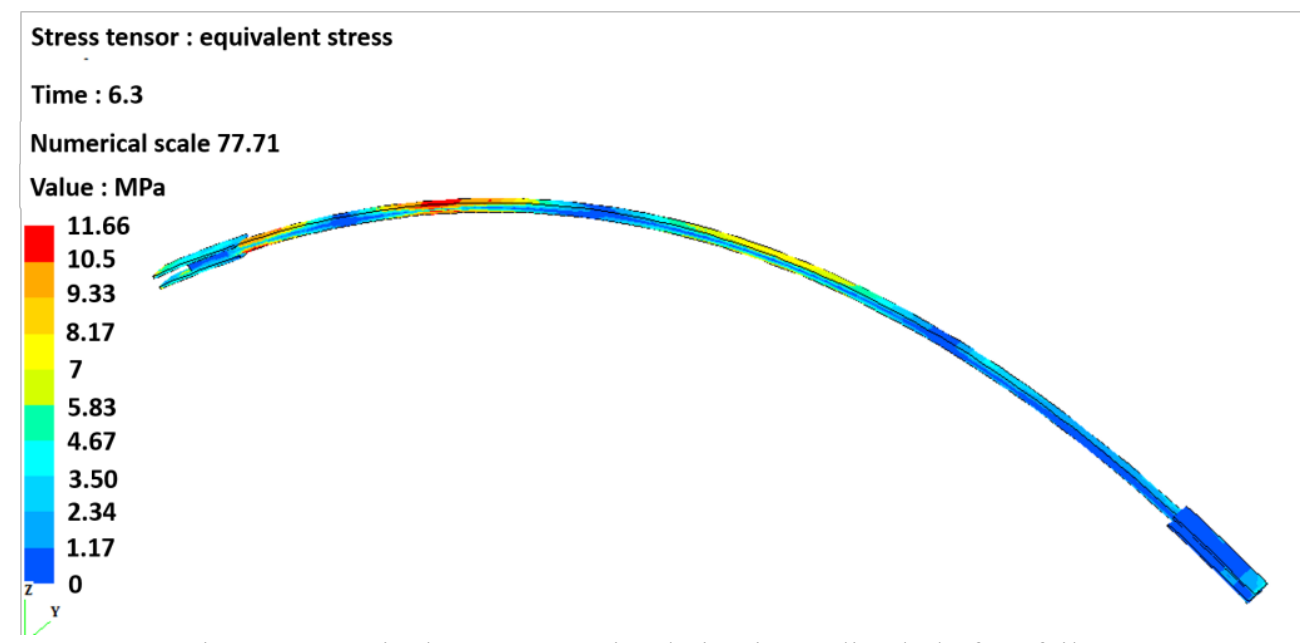

Figure 6. Equivalent stresses simulation immediately before failure.

This also applies to lines 2 and 8 of Table 1, with identical modulus and similar imperfection, the difference between the fixed condition and the weakened spring being $26.3 \%$.

\section{CONCLUSIONS}

From a series of 15 scaled tests on specimens the failure of free standing arches with different torsional stiffness of the arch springs, has been determined. However, other influencing parameters as the E-modulus and initial imperfection showed too many scatter to closely substantiate the influence of the arch torsion stiffness. However, comparing the failure load of similar conditions, the reduction of torsion stiffness by $81.48 \%$ reduces the failure load by $26.3 \%$. This indicates that total prevention of axial rotation may not be imperative for arch bridges and encourages to continue this research, in future by using a material with more constant characteristics.

\section{References}

Van Bogaert, Ph., Schotte, K., and De Backer, H., Post-Failure Torsion Capacity and Robustness of Encased Tubular Arch Spring Connections, $6^{\text {th }}$ IALCCE Symposium Life-cycle Analysis and Assessment in Civil Engineering, Towards an Integrated Vision, Caspeele, Taerwe, Frangopol (eds.), 394-394, ISBN 9781138626331, CRC Press, Ghent, Oct 26-31, 2018.

Van Bogaert, Ph., Torsion Clamping by Prestressing Bars of an Arch Bridge in Concrete Abutment, $38^{\text {th }}$ FIB symposium Engineering a Concrete Future, Modeling and Construction, A. Dancygier (ed.), 447450, ISBN 978659203901, Tel Aviv, Israel. April 22-24, 2013.

William, L. Ko, Accuracies of Southwell and Force/Stiffness Methods in The Prediction of Buckling Strength of Hypersonic Aircraft Wing Tubular Panels, NASA Report N 88-17090, Ames Research Centre, Dryden Flight Facility, Edwards, California USA, November, 1987. 